\title{
Line-Splitting in High-Resolution Superconducting Tunnel Junction EUV Detectors
}

\author{
S. Friedrich, L. J. Hiller, M. F. Cunningham, S. E. Labov
}

This article was submitted to Applied Superconductivity Conference 2000, Virginia Beach, VA, September 17-22, 2000

September 18, 2000

U.S. Department of Energy

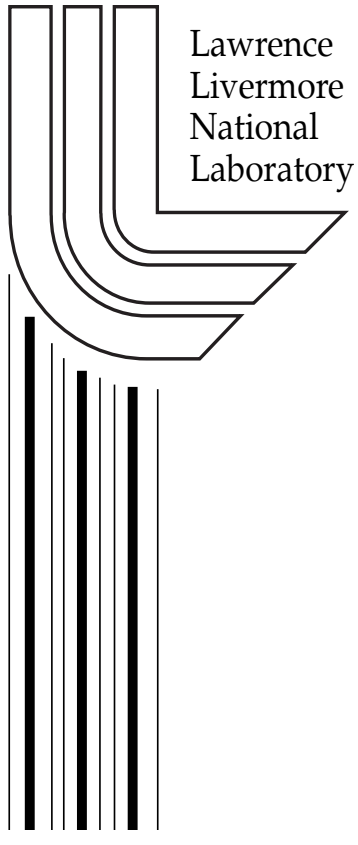




\section{DISCLAIMER}

This document was prepared as an account of work sponsored by an agency of the United States Government. Neither the United States Government nor the University of California nor any of their employees, makes any warranty, express or implied, or assumes any legal liability or responsibility for the accuracy, completeness, or usefulness of any information, apparatus, product, or process disclosed, or represents that its use would not infringe privately owned rights. Reference herein to any specific commercial product, process, or service by trade name, trademark, manufacturer, or otherwise, does not necessarily constitute or imply its endorsement, recommendation, or favoring by the United States Government or the University of California. The views and opinions of authors expressed herein do not necessarily state or reflect those of the United States Government or the University of California, and shall not be used for advertising or product endorsement purposes.

This is a preprint of a paper intended for publication in a journal or proceedings. Since changes may be made before publication, this preprint is made available with the understanding that it will not be cited or reproduced without the permission of the author.

This work was performed under the auspices of the United States Department of Energy by the University of California, Lawrence Livermore National Laboratory under contract No. W-7405-Eng-48.

This report has been reproduced directly from the best available copy.

Available electronically at http://www.doc.gov/bridge

Available for a processing fee to U.S. Department of Energy

And its contractors in paper from

U.S. Department of Energy

Office of Scientific and Technical Information

P.O. Box 62

Oak Ridge, TN 37831-0062

Telephone: (865) 576-8401

Facsimile: (865) 576-5728

E-mail: reports@adonis.osti.gov

Available for the sale to the public from

U.S. Department of Commerce

National Technical Information Service

5285 Port Royal Road

Springfield, VA 22161

Telephone: (800) 553-6847

Facsimile: (703) 605-6900

E-mail: orders@ntis.fedworld.gov

Online ordering: http://www.ntis.gov/ordering.htm

Or

Lawrence Livermore National Laboratory

Technical Information Department's Digital Library

http://www.llnl.gov/tid/Library.html 


\title{
Line-Splitting in High-Resolution Superconducting Tunnel Junction EUV Detectors
}

\author{
Stephan Friedrich, Lawrence J. Hiller, Mark F. Cunningham, and Simon E. Labov
}

\begin{abstract}
We have developed high-resolution Nb-Al-AlOxAl-Nb tunnel junction extreme ultra-violet (EUV) detectors. In the energy range between 25 and $70 \mathrm{eV}$, we have measured an energy resolution of $2.2 \mathrm{eV}$ full-width at half maximum (FWHM). The energy resolution degrades significantly in the energy range between $\approx 80$ and $\approx 230 \mathrm{eV}$ where the $\mathrm{Nb}$ absorber is partially transparent and some of the photons are absorbed in the Al trap layers. We have for the first time observed a distinctly different response for photons absorbed in the $\mathrm{Nb}$ and the Al layer of the same junction electrode. We have modeled this effect with Monte-Carlo simulations of the charge generation process in superconducting multilayers.
\end{abstract}

Index Terms - Energy relaxation, EUV detectors, line shapes, superconducting tunnel junctions

\section{INTRODUCTION}

$\mathrm{S}$ UPERCONDUCting tunnel junctions (STJs) have been developed over the last decade as high resolution energydispersive detectors for photons from optical to $\mathrm{x}$-ray energies [1]. STJ detectors offer single photon efficiency and high energy resolution over a broad band at moderately high count rates.

STJ detectors utilize the small superconducting energy gap to generate about a factor 1000 more excess charge carriers (quasiparticles) upon photon absorption than conventional semiconductor detectors. This translates into a significantly higher energy resolution between $0.12 \mathrm{eV}$ full-width at half maximum (FWHM) at $1 \mathrm{eV} \mathrm{[2]} \mathrm{and} 8.9 \mathrm{eV}$ FWHM at $1 \mathrm{keV}$ [3]. STJ detectors also profit from a comparably short quasiparticle life times of order several microseconds, which allows high-resolution operation at count rates up to 10,000 counts/s [4]. Typical STJ detectors employ superconductors with different energy gaps for quasiparticle trapping to separate absorber and detector functions [5], [6]. Using a thick large gap $\mathrm{Nb}$ or $\mathrm{Ta}$ film for efficient absorption and coupling it to a thin $\mathrm{Al}$ tunnel junction, absorption efficiencies between $60 \%$ and $95 \%$ have been achieved.

One problem in thin-film STJ detectors using vertical

Manuscript received September 19, 2000. This work was performed under the auspices of the U.S. Department of Energy by University of California Lawrence Livermore National Laboratory under contract No. W-7405-Eng48. Funding was provided by the NASA Space Astrophysics Detector Development Program grant NAG 5-4137 and the NASA High Energy Astrophysics Supporting Research and Technology Program under interagency agreement W19.121.

S. Friedrich is a physicist at the Lawrence Berkeley National Laboratory, MS 6-2100, Berkeley, CA 94720, USA. He also works as a participating guest in the Advanced Detector Group at the Lawrence Livermore National Laboratory (telephone: 510-486-7319, e-mail: SFriedrich@lbl.gov).

L. J. Hiller and M. F. Cunningham are graduate students in the Department of Applied Science at the University of California at Davis (email: hiller2@1ln1.gov, cunningham14@1lnl.gov).

S. E. Labov leads the Advanced Detector Group at the Lawrence Livermore National Laboratory, L-418, Livermore, CA 94551, USA (telephone: 925-423-3818, e-mail: labov1@llnl.gov). trapping is that their resolution degrades at photon energies where the absorber film is partially transparent. This is because the bottom electrode of an STJ detector may have a slightly different response function from the top electrode, such that photons absorbed in it cause line broadening or even line splitting. This artifact can be avoided in STJ detectors with lateral trapping [6], which do, however, require a second detector junction for readout and must be operated at lower count rates. The artifact's influence can also be greatly reduced in STJs with a separate heavy metal absorber like $\mathrm{Pb}$ [7]. Most STJ detectors currently used do, however, display some line splitting artifact.

Another question is how much the response function of an STJ detector varies vertically for photons absorbed at different heights within the same junction electrode. This is one possible source of resolution degradation, although recent high-resolution measurements suggest that it can be made negligibly small [2].

Here, we present measurements of the EUV response of $\mathrm{Nb}$-based STJ detectors. We show an unexpected line splitting into at least three lines in response to monochromatic photons in the energy range between 80 and $230 \mathrm{eV}$ where the $\mathrm{Nb}$ absorber is partially transparent. We have modeled the line shape with spatially dependent MonteCarlo simulations of the charge generation process in superconducting bilayers. We will also discuss the relevance of this effect for detector design and performance.

\section{EXPERIMENT}

\section{A. Experimental Setup}

We have developed superconducting Nb-Al-AlOx-Al-Nb EUV and X-ray detectors for high-resolution spectroscopy in astrophysics, material science and biophysics [8], [9]. They consist of a $265 \mathrm{~nm}$ bottom $\mathrm{Nb}$ film and a $50 \mathrm{~nm}$ bottom $\mathrm{Al}$ trap, which is oxidized to form a thin AlOx tunneling barrier, a top $50 \mathrm{~nm} \mathrm{Al}$ trap and a $165 \mathrm{~nm} \mathrm{Nb}$ absorber film. They are fabricated photolithographically on an oxidized Si sub-strate at Conductus Inc. in Sunnyvale, CA [10].

We operate these STJ detectors in a single-stage adiabatic demagnetization refrigerator (ADR) that can be attached directly to a synchrotron beam line. The ADR attains a base temperature below $0.1 \mathrm{~K}$ and a hold time of 8 hours below $0.5 \mathrm{~K}$, the maximum operating temperature of our STJ detectors. After that, a 45-minute magnetization cycle returns the ADR to its base temperature. The ADR temperature does not need to be regulated, as the detector response in temperature independent below $0.5 \mathrm{~K}$.

The low energy data shown here $(25$ to $70 \mathrm{eV})$ were taken at beam line 8-1, the higher energy data (above $60 \mathrm{eV}$ ) at beam line 1-1 of the Stanford Synchrotron Radiation Laboratory (SSRL). For measurements below $70 \mathrm{eV}$, we use three $1000 \AA$ pure $\mathrm{Al}$ windows on a Ni grid to suppress noise 
due to infrared photon absorption and to increase the ADR hold time. At higher energies, we use three $200 \AA \mathrm{Al}$ windows supported by $1000 \AA$ of parylene and a Ni grid.

The photon-induced tunneling current, which provides a measure of the photon energy, is measured with a customdesigned current sensitive preamplifier with a DC voltage bias [11]. The preamplifier signal is processed with a commercial Canberra 2020 amplifier with a shaping time of $3 \mu \mathrm{s}$ and recorded with a Nucleus multichannel analyzer.

\section{B. Experimental Results}

Figure 1 shows a typical EUV spectrum for photon irradiation at $60 \mathrm{eV}$. In addition to the dominant first order line of the grating monochromator at $60 \mathrm{eV}$, higher order contributions at 120,180 and $240 \mathrm{eV}$ can also be seen (fig. 1, top). We observe a clear broadening of the detector response at 120 and $180 \mathrm{eV}$, but hardly at $240 \mathrm{eV}$. This broadening occurs at energies where the $\mathrm{Nb}$ absorber film is partially transparent and a significant fraction of the photons are absorbed in the $\mathrm{Al}$ trap. (The relative absorption efficiencies of the four films of our STJ detector are shown in fig. 1, middle.) This effect has been seen before [4], and it is well understood as being due to a somewhat different detector response from the top and bottom electrode.

One striking and unexpected result is a line splitting into at least three lines, which is most clearly resolved for $180 \mathrm{eV}$ photons (fig. 1, top inset). Comparison of the line intensity ratios with the theoretical absorption efficiencies shows that the two lower-energy peaks are due to photon absorption in the top $\mathrm{Nb}$ and $\mathrm{Al}$ layer, while the higher-energy peak is due to absorption in the $\mathrm{Nb}$ and $\mathrm{Al}$ film of the counterelectrode. This agrees with the observation that the calibration of the spectrum based on the 60 and $240 \mathrm{eV}$ lines, which originate from absorption in the top $\mathrm{Nb}$ film, places the peak assigned to the top $\mathrm{Nb}$ film close to $180 \mathrm{eV}$, while the peaks associated with absorption in the other films deviate from this calibration. It is possible to fit the $180 \mathrm{eV}$ line complex to a four-line multiplet, but this does not improve the quality of the fit because the absorption in the bottom $\mathrm{Nb}$ film is too weak at this energy to be clearly resolved.

The surprising aspect of this line-splitting artifact is that the volume of charge generation upon photon absorption, also known as "hotspot", has been assumed to be much bigger than the film thickness in thin film devices such as ours [12]. Differences in absorption location throughout the $\mathrm{Nb} / \mathrm{Al}$ bilayer should thus have been washed out during the initial charge generation process, since the high-energy quasiparticles and phonons of the hotspot should have spread out evenly through the bilayer well before they relax to low energies. The fact that this is apparently not the case suggests that some elements of the initial charge generation process are not yet completely understood. Below we discuss possible explanations of this line-splitting artifact.

Figure 1 also shows the measured resolution as a function of energy (bottom graph). At energies below $70 \mathrm{eV}$, the resolution is about $2.3 \pm 0.1 \mathrm{eV}$ FWHM, limited by an electronic noise of $2.0 \mathrm{eV}$. At $240 \mathrm{eV}$, the resolution degrades to $4.1 \pm 0.2 \mathrm{eV}$ FWHM because statistical fluctuations in the charge generation and tunneling processes are more important. The resolution is significantly reduced in the intermediate energy range where the $\mathrm{Nb}$ is partially transparent due to line splitting. It varies between 7.6 and $9.5 \mathrm{eV}$ FWHM when fitting the multiplet to a single Gaussian. It can be improved to $4.2 \pm 0.2 \mathrm{eV}$ if the fitting range is limited to just include events due to absorption in the top $\mathrm{Nb}$ layer.
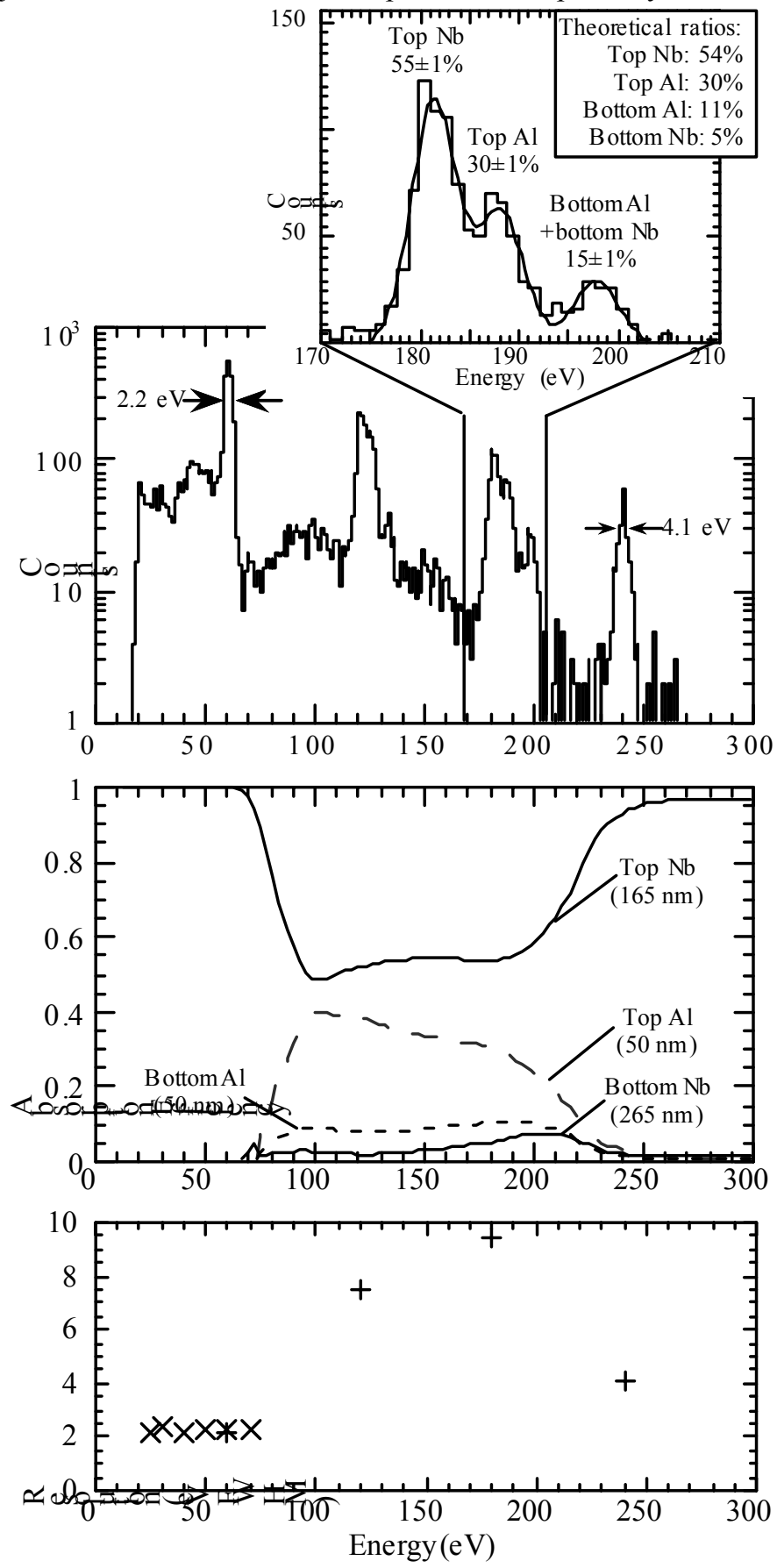

Fig. 1. STJ response to photon irradiation at 60, 120, 180 and $240 \mathrm{eV}$ (top). The inset is an enlargement of the line separation in the region between 170 and $210 \mathrm{eV}$. The middle graph shows the relative absorption efficiencies of the four films of the STJ detector. The bottom graph shows resolution as a function of energy as extracted from a Gaussian fit to the lines in the above spectrum $(+)$ and to a separate set of lower energy measurements $(\times)$. The error bars are smaller than the markers used. 


\section{COMPUTER SiMULATIONS}

\section{Charge Generation Model}

The charge generation process in superconductors in response to photon absorption consists of three phases [12]. The primary photoelectron initially shares its energy through electron-electron interactions until their average energy is comparable to the Debye energy. This process dominates for about 0.1 to 1 ps depending on the superconductor and produces electrons with average energy around $0.1 \mathrm{eV}$. At that point electron-phonon scattering becomes a faster relaxation mechanism. Most electrons will thus relax by phonon emission, and for a period of about 10 to 100 ps the hotspot expands with the phonon velocity. Finally, phonons with energy above the superconducting gap $2 \Delta$ will break Cooper pairs, thereby generating a measurable number of excess quasiparticles. These quasiparticles continue to relax by phonon emission and phonons will continue to break Cooper pairs until all phonon energies are below 2 $\Delta$. Such sub-gap phonons can no longer break Cooper pairs and will eventually escape into the substrate. The entire energy relaxation process takes about 1 ns.

Most Monte-Carlo simulations of this process are only concerned with the average energy required to generate excess quasiparticles and the associated statistical fluctuations, which determine the ultimate resolution limit of STJ detectors [13]. Therefore, they only have to consider a homogeneous superconductor, which in turn eliminates the need to simultaneously model energy relaxation and diffusion, thereby greatly reducing computational requirements. The line-splitting artifact shows that this assumption is obviously no longer satisfied in the multilayer devices discussed here.

\section{Monte-Carlo Simulations}

We have performed Monte-Carlo simulations of the energy relaxation process upon photon absorption in heterogeneous superconducting multilayers [14].

Details of the initial electron-electron interaction have little relevance for the charge generation, since any information on the resulting electron distribution is lost during the subsequent cascade. We thus start our simulation with an initial set of phonons randomly distributed between zero and the Debye energy with a total energy equal to the energy of the incident photon. Each phonon with energy above $2 \Delta$ is assumed to break a Cooper pair into two quasiparticles. The energies of these two quasiparticles are randomly selected, subject to energy conservation and the constraint that the average probability to create a quasiparticle with a certain energy agree with theory [15]. Each quasiparticle is then allowed to relax to a random lower energy, again constrained by energy conservation and an average relaxation probability to a certain lower energy given by theory [15]. These two processes are repeated until no phonons with energy above $2 \Delta$ are left and no more Cooper pairs can be broken. The total number of quasiparticles is then recorded.

The electron mean free path is $\approx 10 \mathrm{~nm}$ in our $\mathrm{Nb}$ and (neglecting surface scattering) $\approx 60 \mathrm{~nm}$ in our Al films, which have a residual resistance ratio of $\approx 4$. The electrons will thus undergo many elastic scattering events before scattering inelastically, thereby randomizing their vertical position in the $\mathrm{Nb} / \mathrm{Al}$ bilayer between inelastic scattering events. We thus assume the electrons are evenly distributed in the bilayer and assume that scattering occurs according to the fraction of time spent in each film and the relative inelastic scattering time at the energy of the electron.

Phonons, on the other hand, have a much lower velocity and a finite transmission probability across layer interfaces [16]. In the Monte-Carlo simulation we therefore keep track of the phonon position and include reflection at the interface between dissimilar materials as well as phonon loss into the oxidized Si substrate.

The results of this simulation are summarized in table 1. The charge generated by photon absorption in the top $\mathrm{Nb}$ absorber is set to unity, and the charge yield from photon absorption in the other layers is normalized relative to it. Within this model, the predicted charge yield from the lower layers of the STJ detector is less than the yield from the top $\mathrm{Nb}$ layer, because high energy phonons from the lower layers are more likely to escape into the Si substrate during the relaxation process. We also note that the charge yields from photon absorption in the $\mathrm{Nb}$ and $\mathrm{Al}$ film of the same junction electrode are very similar, because quasiparticle transmission across the interface is assumed to be high. The results of this simulation do not agree with our observations.

\section{E. Line-Splitting Simulations}

We have modified our Monte-Carlo simulations to assess possible causes of the observed line splitting of $3.7 \%$ between peaks originating from the top $\mathrm{Nb}$ and $\mathrm{Al}$ layers. This separation indicates that the quasiparticles (and also phonons) are not entirely free to move between the layers. In the extreme case where the charge generation process is entirely confined to the absorbing layer, the $\mathrm{Nb}$ and $\mathrm{Al}$ peaks would have a ratio of the energy gaps of 2.2. The observed line splitting suggests that a much more moderate form of confinement is present.

We examine three possible mechanisms to account for the line splitting. The first is an unexpectedly small diffusion constant, which has been observed in Ta and Al-based STJ detectors before [17]. The mean free path of $\approx 60 \mathrm{~nm}$ in our $\mathrm{Al}$ films is comparable to the $50 \mathrm{~nm}$ thickness of the $\mathrm{Al}$ trapping layer, and the corresponding elastic quasiparticle scattering time is 10 to 100 times faster than the inelastic scattering time of moderate- to high-energy quasiparticles. An impurity concentration high enough to cause confinement would be readily apparent in residual resistance ratio measurements, but this is not observed.

A second quasiparticle confinement mechanism would be a reduced quasiparticle transmission across the Al-Nb interface. The transmission due to Fermi velocity mismatch is estimated to be $85 \%$ [18]. It may be further decreased by the accumulation of residual gases on the Al trap surface during fabrication. However, the long mean free path in our Al films implies repeated attempts of the quasiparticles to cross the interface between inelastic scattering events, so confinement would be possible only with a rather small transmission coefficient. We do not believe this to be the case, since the gap values of our proximitized Al layers are well described by the Golubov model of the proximity effect 
[18], which assumes good quasiparticle transmission across the $\mathrm{Nb} / \mathrm{Al}$ interface, rather than the low-transmission McMillan model.

A third mechanism to account for the line splitting is a combination of some confinement plus quasiparticle loss in $\mathrm{Nb}$. We observe that the top $\mathrm{Nb}$ layer generates smaller signals than the bottom $\mathrm{Nb}$ layer, despite the tendency for the bottom layer to lose phonons into the substrate. One possible loss process is quasiparticle trapping in $\mathrm{Nb}$ oxides. A Nb film typically forms $a \approx 6 \mathrm{~nm}$ oxide layer at an exposed surface, parts of which consist of metallic $\mathrm{NbO}$ [19]. This metallic surface layer can effectively trap quasiparticles and thus reduce the charge yield of the top $\mathrm{Nb}$ electrode relative to the bottom. However, it cannot account for the line splitting of peaks between $\mathrm{Nb}$ and $\mathrm{Al}$ layers of the same electrode. Some form of quasiparticle confinement is also required to shield quasiparticles in the $\mathrm{Al}$ from being lost in the $\mathrm{Nb}$ film.

We have modified our multilayer Monte Carlo simulation to examine this third mechanism. We assume that a certain fraction of the quasiparticles relaxing in the top $\mathrm{Nb}$ layer is completely lost into the metallic $\mathrm{NbO}$ layer. We also simulate confinement by not letting quasiparticles change layers between inelastic scattering events whenever their energy dependent inelastic scattering time $\tau_{\text {in }}(\mathrm{E})$ is shorter than some multiple of the average transit time $2 \mathrm{~d} / \mathrm{v}_{\mathrm{Fermi}}$ they would require to traverse the film of thickness $d$ ballistically. This ballistic approximation of diffusive motion is crude, but reduces the computational requirement considerably.

These two values were adjusted to produce the observed multiplet of figure 1. A close approximation was obtained with a loss fraction of $9.2 \%$ and a confinement condition of $\tau_{\text {in }}(\mathrm{E})<2.7 \mathrm{~d} / \mathrm{v}_{\text {Fermi. }}$. This means that the hotspot is confined to the layer of photon absorption until the quasiparticles have relaxed to an average energy of $\approx 18 \mathrm{meV}$. It also means that charge loss into the $\mathrm{Nb}$ oxide layer reduces the signal from events in the top $\mathrm{Nb}$ layer by $19.4 \%$. The signal from events in the top Al layer is only reduced by $14.8 \%$. The loss process has a negligible effect on charge yield from both layers of the bottom electrode. This simulation also places the weak peak originating from absorption events in the bottom $\mathrm{Nb}$ film at $194.1 \mathrm{eV}$ (cf. fig. 1).

TABLE I

CHARGE Yield IN EXPERIMENT AND SiMULATIONS

\begin{tabular}{|c|c|c|c|c|}
\hline Layer & $\begin{array}{l}\text { Measur } \\
(\mathrm{eV})\end{array}$ & $\begin{array}{l}\text { Signal } \\
(\%)\end{array}$ & $\begin{array}{c}\text { Simulat } \\
\text { No conf./loss } \\
(\%) \\
\end{array}$ & $\begin{array}{l}\text { ted Signal } \\
\text { With conf./loss } \\
(\%) \\
\end{array}$ \\
\hline Top $165 \mathrm{~nm} \mathrm{Nb}$ & 181.4 & 100 & 100 & 100 \\
\hline Top $50 \mathrm{~nm} \mathrm{Al}$ & 188.2 & 103.7 & 99.5 & 104.3 \\
\hline Bottom $50 \mathrm{~nm} \mathrm{Al}$ & 198.8 & 109.6 & 92.6 & 108.2 \\
\hline $\begin{array}{l}\text { Bottom } 265 \mathrm{~nm} \\
\mathrm{Nb}\end{array}$ & \multicolumn{2}{|c|}{ not resolved } & 91.5 & 107.0 \\
\hline
\end{tabular}

The results of this simulation, normalized to the charge yield of the top $\mathrm{Nb}$ film, are summarized in the right column of table 1. Despite the crude approximations, the basic features of the multiplet are correctly identified using reasonable assumptions regarding quasiparticle loss and confinement. While metallic surface oxides constitute one possible loss mechanism in $\mathrm{Nb}$, the simulation would also correctly describe other loss process like trapped flux or grain boundaries with reduced energy gap. More detailed MonteCarlo simulations would model the diffusive quasiparticle motion during the energy relaxation process as a random walk and include the finite transparency of the $\mathrm{Nb} / \mathrm{Al}$ interface.

\section{SUMMARY}

We have developed high-resolution superconducting NbAl-AlOx-Al-Nb tunnel junction EUV detectors. At energies below $70 \mathrm{eV}$, they have a quantum efficiency close to unity and an energy resolution of $2.3 \pm 0.1 \mathrm{eV}$ FWHM, limited so far by the electronic noise contribution of the preamplifier. In the energy range between 80 and $230 \mathrm{eV}$, where the $\mathrm{Nb}$ absorber film is partially transparent, we observe a linesplitting artifact of at least three distinct lines in response to monochromatic photons due to photon absorption in the $\mathrm{Al}$ trap layers. We have extended our Monte-Carlo simulations of the charge generation process in heterogeneous superconducting multilayers to simulate this line splitting between the $\mathrm{Nb}$ and the $\mathrm{Al}$ film of the same junction electrode. They suggest that this artifact is due to quasiparticle confinement during the initial stage of the charge generation process combined with quasiparticle loss in the top $\mathrm{Nb}$ absorber film.

\section{REFERENCES}

[1] For a recent overview of cryogenic detector research see "Proc. 8th Intl. Workshop on Low-Temperature Detectors (LTD-8)", Nucl. Inst. Meth., vol. A444, 2000

[2] P. Verhoeve, "UV/optical imaging spectroscopy with cryogenic detectors", Nucl. Inst. Meth. A, vol. 444, pp.435-440, 2000

[3] J.B. LeGrand et al., "A superconducting tunnel junction X-ray detector with performance limited by statistical effects", Appl. Phys. Lett., vol. 73, pp. 1295-1297, 1998

[4] M. Frank et al., "Energy resolution and high count rate performance of superconducting tunnel junction x-ray spectrometers", Rev. Sci. Inst., vol. 69 , pp. 25-31, 1998

[5] N.E. Booth, "Quasiparticle trapping and the quasiparticle multiplier", Appl. Phys. Lett., vol. 50, pp. 293-295, 1987

[6] H. Kraus, F. v. Freilitzsch, J. Jochum, R.L. Mössbauer, T. Peterreins, F. Pröbst, "Quasiparticle trapping in a superconductive detector system exhibiting high energy and position resolution", Phys. Lett. B vol. 231, pp. 195-202, 1989

[7] G. Angloher et al., submitted for publication to Appl. Phys. Lett., 2000

[8] M. Frank et al., "Cryogenic high-resolution X-ray spectrometers for SRXRF and microanalysis", J. Synchrotron Rad., vol. 5, pp. 515-517, 1998

[9] S. Friedrich et al., "Superconducting high-resolution X-ray detectors for metalloprotein L-edge spectroscopy", J. El. Spectr. and Rel. Phenomena, vol. 101, pp. 891-896, 1999

[10] A.T. Barfknecht, R.C. Ruby, H. Ko, "A simple and robust niobium Josephson junction integrated circuit process", IEEE Trans. Magn., vol. 27, pp. 3125-3128, 1991

[11] S. Friedrich et al., "Single photon imaging X-ray spectrometers using low noise current preamplifiers with dc voltage bias", IEEE Trans. Appl. Superconductivity, vol. 7, pp. 3383- 3386, 1997

[12] A. Zehnder, "Response of superconductive films to localized energy deposition", Phys. Rev. B., vol. 52, pp. 12858-12866, 1995

[13] N. Rando et al., "The properties of $\mathrm{Nb}$ superconducting tunneling junctions as X-ray detectors", Nucl. Inst. Meth. A, vol. 313, 173-195 (1992)

[14] L.J. Hiller, "Applications of superconducting tunnel junctions to X-ray fluorescence measurements", Dissertation, University of California at Davis, 2000; L.J. Hiller, to be published. 
[15] S.B. Kaplan, C.C. Chi, D.N. Langenberg, J.J. Chang, S. Jafarey, D. J. Scalapino, "Quasiparticle and phonon lifetimes in superconductors" Phys. Rev. B, vol. 14, pp. 4854-4873, 1976

[16] S.B. Kaplan, "Acoustic matching of superconducting films to substrates", J. Low Temp. Phys., vol. 37, pp. 343-365, 1979

[17] S. Friedrich, K. Segall, M.C. Gaidis, C.M. Wilson, D.E. Prober, A.E. Szymkowiak, S.H. Moseley, "Experimental quasiparticle dynamics in a superconducting imaging X-ray spectrometer", Appl. Phys. Lett., vol. 71, pp. 3901-3903, 1998; S. Friedrich, "Superconducting single photon imaging X-ray spectrometers", Dissertation, Yale University, 1997

[18] A.A. Golubov et al., "Proximity effect in superconductor-insulatorsuperconductor Josephson tunnel junctions: theory and experiment", Phys. Rev. B, vol. 51, pp. 1073-1089, 1995

[19] M. Grundner, J. Halbritter, "XPS and AES studies on oxide growth and oxide coatings on niobium", J. Appl. Phys., vol. 51, pp. 397-405, 1980 